\title{
A sub-millimetric, $0.25 \mathrm{mN}$ resolution fully integrated fiber-optic force-sensing tool for retinal microsurgery
}

\author{
Iulian Iordachita - Zhenglong Sun - Marcin Balicki • \\ Jin U. Kang • Soo Jay Phee · James Handa • \\ Peter Gehlbach • Russell Taylor
}

Received: 10 January 2009 / Accepted: 18 March 2009 / Published online: 15 April 2009

(C) CARS 2009

\begin{abstract}
Purpose Retinal microsurgery requires extremely delicate manipulation of retinal tissue where tool-to-tissue interaction forces are usually below the threshold of human perception. Creating a force-sensing surgical instrument that measures the forces directly at the tool tip poses great challenges due to the interactions between the tool shaft and the sclerotomy opening.

Methods We present the design and analysis of a force measurement device that senses distal forces interior to the sclera using 1-cm long, $160 \mu \mathrm{m}$ diameter Fiber Bragg Grating (FBG) strain sensors embedded in a $0.5 \mathrm{~mm}$ diameter tool shaft. Additionally, we provide an algorithm developed to cancel the influence of environmental temperature fluctuations.

Results The force-sensing prototype measures forces with a resolution of $0.25 \mathrm{mN}$ in 2 DOF while being insensitive to temperature.

Conclusion Sub-millinewton resolution force sensors integrated into microsurgical instruments are feasible and
\end{abstract}

I. Iordachita $(\varangle)$

Johns Hopkins University, 3400 N. Charles Street,

CSEB 112, Baltimore, MD 21218, USA

e-mail: iordachita@jhu.edu

I. Iordachita $\cdot$ Z. Sun $\cdot$ M. Balicki $\cdot$ J. U. Kang $\cdot$ R. Taylor

ERC for Computer Integrated Surgery,

Johns Hopkins University, Baltimore, MD, USA

Z. Sun · S. J. Phee

Robotic Research Center, Nanyang Technological University,

Singapore, Singapore

J. Handa $\cdot$ P. Gehlbach

Wilmer Eye Institute, Johns Hopkins Hosptial,

Baltimore, MD, USA have potential applications in both robotic and freehand microsurgery.

Keywords Microsurgery · Force sensor - Surgical instruments · Computer-assisted surgery

\section{Introduction}

Many clinical procedures involve intervention and manipulation of extremely small, delicate tissue structures. Retinal microsurgery is an example of the requirement for micronlevel maneuvers. The manipulation of vitreoretinal structures inside the eye poses enormous challenges, due to tissue delicacy, surgical inaccessibility, suboptimal visualization, and the potential for irreversible tissue damage resulting from unintentional movement.

In current practice, retinal surgery is performed under a surgical microscope. Small (20-25 gauge) surgical instruments are inserted through the sclera of the eye through operative sclerotomy sites (typically, 2-3). The main technical limitations in vitreoretinal surgery are:

(1) inadequate spatial resolution and depth perception of microstructures to identify tissue planes,

(2) imprecise movements during micromanipulation of tissue due to physiological tremor. Physiological tremor contributes to increases in operative time is exacerbated by fatigue, and is a significant limiting factor in microsurgery [1].

(3) lack of force sensing since the movements required for dissection are below the surgeon's sensory threshold. Gupta et al. [2] reported that a majority of retinal surgery is performed without force sensation of the interactions between retinal tissue and the surgical tool. 
These factors collectively not only make vitreoretinal surgery the most technically demanding ophthalmologic surgery, but also apply similarly to other microsurgical disciplines such as otolaryngology, vascular surgery as well as neurosurgery. At Johns Hopkins University, we have had a long-standing research program intended to address these limitations. The present program utilizes the steady-hand "Eye Robot" [3,4] and various "sensory substitution" schemes to provide the surgeon with feedback on tool-to-tissue forces that would otherwise be imperceptible [5].

There have been several investigations of force sensing for microsurgery over the years. For example, Zhou et al. [6] developed a force-sensing scheme relying on measuring the deflection of an optical beam. Kim et al. [7] developed multiaxis MEMS force sensors in an approximately $3 \times 5 \times 0.5 \mathrm{~mm}$ form factor. Menciassi et al. [8] developed a 15.5-mm microgripper with integrated strain gauge sensors. Early work by Gupta et al. [2] included use of a 1 DOF pick-like probe to measure forces in retinal surgery and to explore the feasibility of a simple auditory "sensory substitution" scheme to assist the surgeon in controlling these forces. They determined that $75 \%$ of these forces were less than $7.5 \mathrm{mN}$ in magnitude and that only $19 \%$ of force events of this magnitude are felt by the surgeon. Subsequently, Berkelman et al. developed a 3 DOF force sensor $[9,10]$ for use in ENT and eye applications with the JHU "Steady Hand" microsurgery system. Jagtap and Riviere [11] incorporated this sensor into a hand-held instrument and used it to measure forces in retinal tasks both on cadaveric pig retinas and in vivo rabbit eyes. Experience with this instrument shows that in vivo measurements are indeed feasible, but that discrimination between forces applied at the tool tip and forces from contact with the sclera may be a challenge if the force sensing is done distal to the sclerotomy point. The difficulty is not so much friction between the tool shaft and sclerotomy opening as it is lateral forces exerted on the tool shaft during tool manipulation.

These prior approaches pose a number of limitations to retinal microsurgery application. With the exception of [2], which used a strain gauge along the tool shaft, the sensor "packages" are too large to be incorporated easily into the portion of the microsurgical tool that is inside the eye. Placing a multi-axis force sensor in the tool handle, as was done in $[9,10]$ necessarily introduces significant sclera-to-tool force disturbances that can completely swamp the tool-to-tissue force measurements. Fabricating custom micro-MEMS sensors into the actual tool tip (e.g., into a $0.5 \mathrm{~mm}$ cutter blade or tweezers tip) is conceptually possible, but poses numerous fabrication, assembly, and interfacing problems.

These considerations led us to explore approaches in which sensors could be mounted along the portion of the tool shaft inside the eye. We looked at several sensing technologies, including conventional strain gauges, but settled on Fiber Bragg Grating (FBG) strain sensors. Optical fiber sensors are small (75-200 $\mu \mathrm{m}$ diameter), extremely precise, mechanically stable, immune to electrical noise, sterilizable, relatively inexpensive, and can be built into a $\sim 1 \mathrm{~mm}$ diameter tool. In addition, expertise gained at integrating FBGs into our tools could be readily transferred to other fiber-optic sensors and devices for microsurgery, as well as into other robotic and sensing applications in our laboratory.

In subsequent sections, we will describe the functional requirements and conceptual design for our force-sensing tools. After presenting preliminary validation experiments with a 1 DOF prototype, we will describe the design of a temperature-insensitive 2 DOF tool family, with calibration test results showing its performance. Finally, we will present initial results using a 2 DOF hook tool on a membrane peeling phantom.

\section{Materials and methods}

Tool functional requirements and conceptual design

The design of the force-sensing surgical instrument has to meet both form factor constraints and measurement resolution requirements. The geometry of the surgical environment inside of the eye dictates the sensing element location, which should be close to the distal end of the tool. The sensor should not compromise the overall size of the instrument, and the device should have the capability of measuring forces at the tip with sub-millinewton resolution. Detailed functional specifications are listed in Table 1.

The conceptual design of the force-sensing tool is shown in Fig. 1. As discussed in the introduction, our design approach relies on FBG strain sensors. FBG technology has been used in a number of important application areas ranging from structural monitoring to chemical sensing [12]. Bragg sensors consist of a grating formed inside of a photosensitive optical fiber by exposure to an intense optical interference pattern, which effectively creates a wavelength specific dielectric mirror inside of the fiber core. This characteristic Bragg wavelength shifts due to modal index or grating pitch change from physical deformation caused by strain or temperature change. The fibers themselves have very small diameters $(160 \mu \mathrm{m}$ in our current application), are immune to electrical noise, can be sterilized in various ways, and have excellent biocompatibility characteristics.

Table 1 Design specifications for the force-sensing tool

\begin{tabular}{ll}
\hline Tool shaft diameter & $<1 \mathrm{~mm}$ \\
Tool shaft length & $>30 \mathrm{~mm}$ \\
Force resolution at tip & $\sim 0.25 \mathrm{mN}$ \\
Sampling rate & $>100 \mathrm{~Hz}$ \\
\hline
\end{tabular}



design of optical fiber force-sensing tool; (right) force coordinate system at the tip of the tool
Fig. 1 (Left) Conceptual

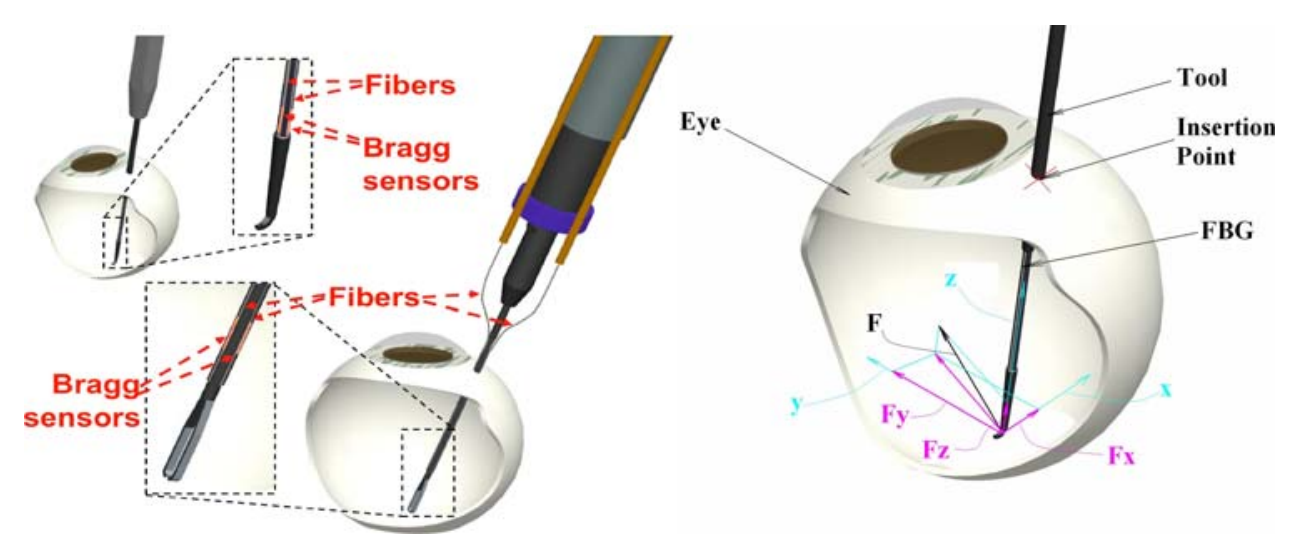

Fig. 2 1-DOF force-sensing tool prototype: cross-section diagram and lateral view
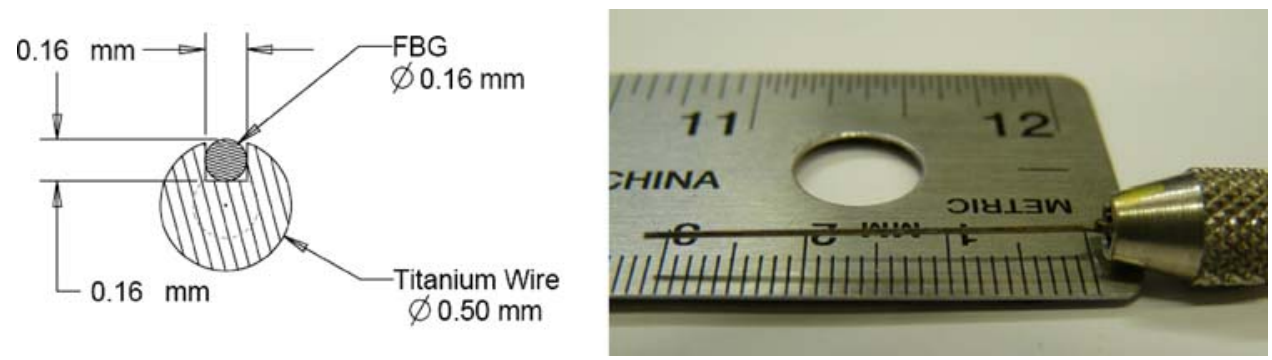

Ultimately, we desire to measure $3 \mathrm{DOF}$ forces resolved at the tip of the surgical instrument. To achieve this, we currently plan to measure translational forces along the tool shaft by embedding 1 DOF force sensing in the tool handle and to place FBG strain sensors along the tool shaft distal to the sclerotomy point. We will use redundant FBG sensors to compensate for errors due to temperature effects, in much the same manner as might be done with conventional electrical strain gauges.

\section{DOF force-sensing prototype}

\section{Design and fabrication}

To mimic 25 gauge ophthalmic instruments, a 50-mm long titanium wire with $0.5 \mathrm{~mm}$ diameter was prepared as the tool shaft. Titanium was selected to provide the tool the necessary toughness and flexibility to allow maximal strain and resulting increased sensitivity. To integrate the FBG optical fiber into the shaft, a square section channel $(160 \times 160 \mu \mathrm{m})$ was machined into the surface along shaft's axial direction. The FBG sensor used here is OS110, from Micron Optics, Inc. (Atlanta, GA), with a central wavelength of $1550 \mathrm{~nm}$. The active fiber section of the sensor is about 10-mm long, starting $5 \mathrm{~mm}$ from the tip of the fiber pigtail. An optical sensing interrogator, sm130-700 from Micron Optics Inc. (Atlanta GA), was used to monitor the sensor. The wavelength interrogator has a resolution of $0.001 \mathrm{~nm}$ and scan frequency up to $2 \mathrm{kHz}$, with four channels.

The cross-sectional profile of a single FBG sensor and a picture of the prototype are shown in Fig. 2. With such a design, the overall size and length of the tool both can meet the design criteria. Calibration testing was performed to determine the force-wavelength relationship and the force measurement resolution.

\section{Force resolution validation}

Calibration was carried out in an electrically shielded analytical balance, Sartorius 1601 from Data Weighing Systems, Inc. (Elk Grove IL), which has a readability of $0.1 \mathrm{mg}$. An acrylic placeholder was placed on the balance below the tool tip. The tool was held horizontally by a pin vise, which was attached to a 3-DOF linear translation stage. The tool was oriented so that the channel with the FBG sensor was either facing upwards or downwards in vertical plane. The tool shaft axis was positioned to be perpendicular to the placeholder's apex. The setup can be found in Fig. 3 .

Through fine adjustment of the height of the vertical translation stage, the force exerted on the shaft tip can be calculated from the weight read from the analytical balance. The resolution of the balance is $0.1 \mathrm{mg}$, which is equivalent to approximately $0.001 \mathrm{mN}$ force for this setup. For every increment of $25 \mathrm{mg}$ in weight, the corresponding wavelength of the FBG sensor was recorded. Calibration was performed 


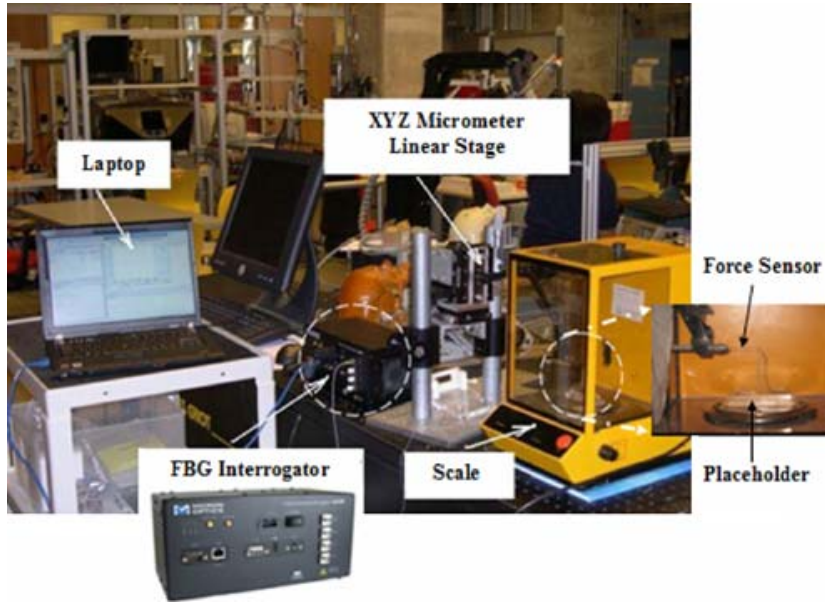

Fig. 3 1DOF force sensor system and calibration setup

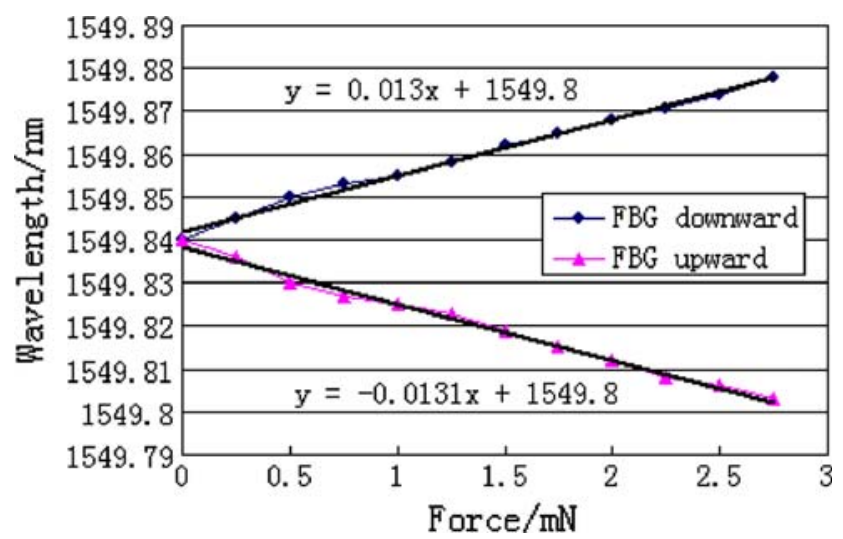

Fig. 4 Calibration results for $1 \mathrm{DOF}$ force-sensing prototype

first with the FBG facing downward and second with the FBG facing upward. By changing the orientation, the surface with the embedded FBG will experience either tension (FBG downward) or compression (FBG upward). The result is shown in Fig. 4. It is notable that the results show a linear relationship between the force exerted and the wavelength in both cases, and that the two calibration curves are almost symmetrical.

From the calibration results, we conclude that the force resolution is adequate to meet the design goal of $0.25 \mathrm{mN}$. Our design has met all of the fundamental design criteria to enable us to create a 2-DOF force-sensing tool.

\section{DOF force-sensing tool}

\section{Design of the 2 DOF force sensor}

To measure the force in the transverse plane (perpendicular to $Z$ axis of the tool shaft, see Fig. 1), three FBG sensors are embedded evenly along the surface of the wire, $120^{\circ}$ from one another. With such a design, the cross section is symmetric

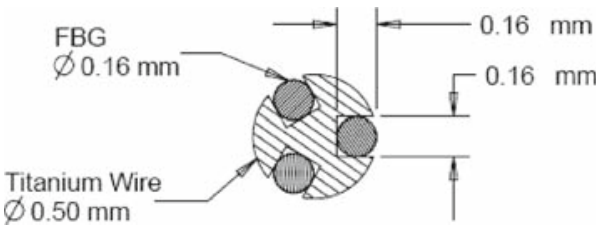

Fig. 5 Cross-section diagram of 2-DOF force-sensing tool

as shown in Fig. 5; the neutral surface is perpendicular to the applied force and passes through the center of the circle.

It is known that the sensitivity of the FBG sensor is governed by the nature of the load or strain which is applied to the structure the fiber is attached to or embedded within [12]. With a force applied at the tip of the wire, the stress on the cross section of the wire can be described by

$\sigma=\frac{y}{R} \sigma_{\max }=\frac{M y}{I}=\frac{F L y}{I}$

where $y$ is the distance from the surface to the neutral surface, $R$ is the radius of the wire, $I$ is the moment of inertia of the wire cross section, $M$ is the moment at the tip, $L$ is the length of the cantilevered shaft, and $F$ is the applied force at the tip. With fixed $L$ and $I$, the strain on the surface of the wire is proportional to the applied force; then the applied force can be calculated from the stress distribution in the cross section.

$\varepsilon=\sigma / E=K \cdot F_{\text {applied }}$.

\section{Force computation algorithm}

To calculate the lateral force components $\left(F_{X}\right.$ and $F_{Y}$ in Fig. 1) an algorithm is developed to eliminate the axial component and provide cancellation of temperature effect. The shift in Bragg wavelength with strain and temperature can be expressed as:

\section{$\Delta \lambda=k_{\varepsilon} \varepsilon+k_{T} \Delta T$}

We assume that there is negligible temperature gradient along the surface of such small volume, so three FBG sensors experience the same $\Delta T$ and the temperature sensitivity $K_{T}$ should be constant for the same type of FBG sensors.

Here we introduce a new parameter, $\Delta S$ as the sensor reading, which is defined as

$$
\Delta S_{i}=\Delta \lambda_{i}-\Delta \lambda_{\text {mean }}=k_{\varepsilon i} \varepsilon_{i}-\frac{1}{3} \sum_{i=1}^{3} k_{\varepsilon i} \varepsilon_{i}
$$

By subtracting the mean value from each wavelength shift, the common terms such as noises, axial strain and the temperature component can be removed. From the expression, we could expect a linear relationship between the sensor reading 
$\Delta S$ and the applied force in corresponding $x-y$ coordinates as shown below:

$\left[\begin{array}{l}\Delta S_{1} \\ \Delta S_{2} \\ \Delta S_{3}\end{array}\right]=\left[\begin{array}{ll}K_{1 x} & K_{1 y} \\ K_{2 x} & K_{2 y} \\ K_{3 x} & K_{3 y}\end{array}\right]\left[\begin{array}{l}F_{X} \\ F_{Y}\end{array}\right]$.

\section{Experimental setup and calibration}

The calibration setup is similar to the previous one, with inclusion of an additional rotary stage to provide an accurate angle adjustment with a resolution of $2^{\circ}$ : Fig. 6 . The coordinate origin was set at the tip of the wire and at the center of the cross section. Initial sensor position with one of the FBG fibers (channel 1) facing up was set to be the $x$ axis. The calibration was performed by rotating the sensor about its long axis 90 degrees and loading the sensor in $0.25 \mathrm{mN}$ steps. Four calibration sets were performed at $0,90,180,270^{\circ}$. Another set of calibrations was performed at $120^{\circ}$ intervals for consistency and symmetry.

The following calibration matrix was obtained:

$K=\left[\begin{array}{cc}-0.014 & -0.0018 \\ 0.00512 & 0.01356 \\ 0.009 & -0.01175\end{array}\right]$

The force then can be calculated using the sensor readings $\Delta S$ and the pseudo-inverse of the calibration matrix:

$\vec{F}=K^{+} \Delta S$.

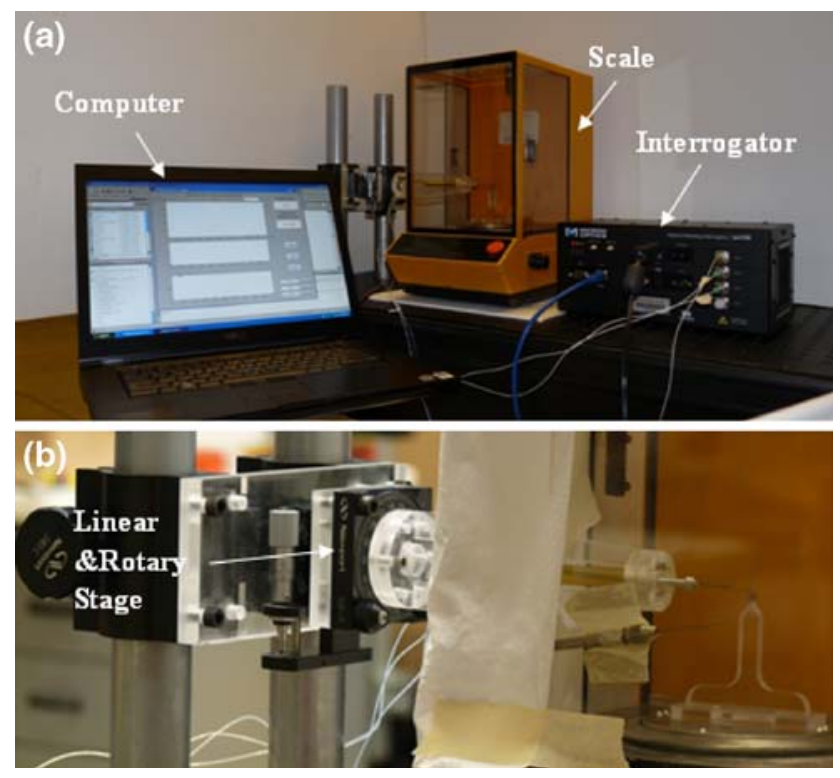

Fig. 6 a Experiment setup for 2 DOF force sensor and $\mathbf{b}$ detail inside the scale

\section{Results}

Temperature compensation

To evaluate the behavior of the 2 DOF force sensor in temperature varying environment, a candle was placed in the enclosure of the scale as a heat source. In the top graph in Fig. 7, the wavelength shifts for three channels are plotted in blue, red and black over time. The wavelength shifts are the same for each FBG sensor when exposed to the same temperature without any loading. A dramatic wavelength shift change can be observed at time $12 \mathrm{~s}$, while the calibrated force components in $x$ - and $y$-axes remain at zero, as observed in the corresponding bottom two graphs. The maximum shift in wavelength due to temperature effect is about 20 picometers, which is equivalent to approximately $2 \mathrm{mN}$ based on previous calibration results.

Testing was also done with the load at an arbitrary angle. By adjusting the linear and rotary stages, we changed the force exerted at the tip from 3.25 to $6.33 \mathrm{mN}$ at a direction of about $70^{\circ}$ from $x$ axis. The data collected and calculated force and angle are shown in Fig. 8.

For the first $12 \mathrm{~s}$, the force was kept at $3.25 \mathrm{mN}$, and then increased to $6.33 \mathrm{mN}$ by moving the linear stage to apply a load on the sensor. A candle was placed near the sensor to increasing the temperature which is represented by a peak in wavelengths shift of all FBGs around 50-60 s. However, the calculated force is unaffected and the angle calculated based on $F_{X}$ and $F_{Y}$ is around 70 degrees during the whole process. It can be concluded that the calibration matrix obtained
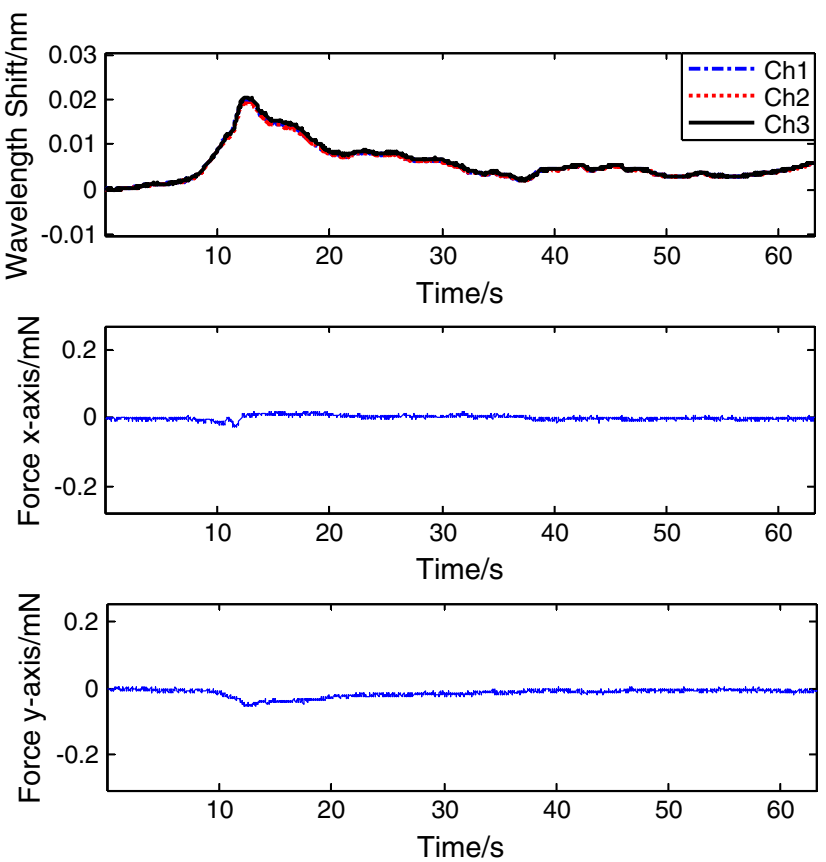

Fig. 7 Temperature compensation test without load 

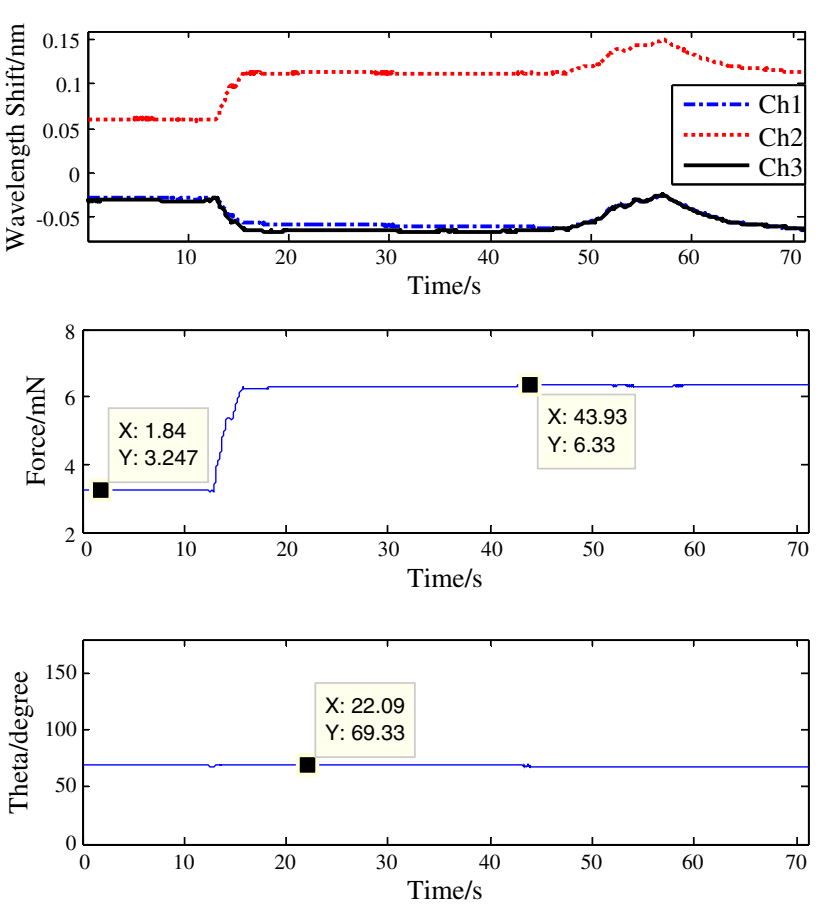

Fig. 8 Temperature compensation test with load

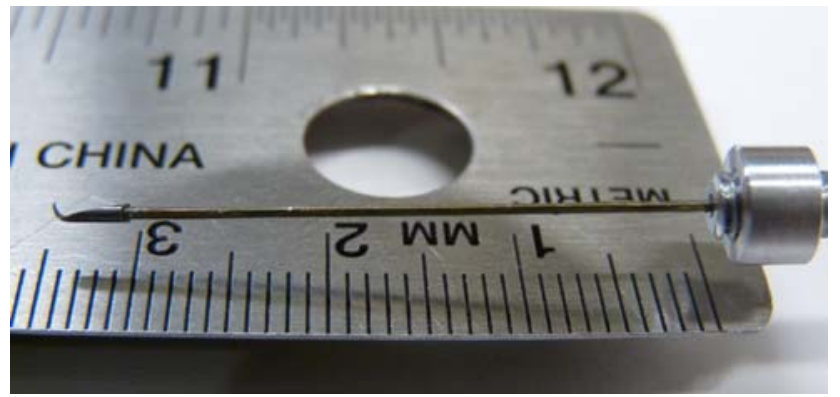

Fig. 9 Customized hook

works very well to evaluate the 2-D force at the wire tip; and the algorithm removes the temperature influence effectively.
Hook instrument with force sensing

Membrane peeling is a clinical task used in the surgical treatment of epiretinal membrane (ERM). Epiretinal membrane is a thin (i.e. $5 \mu \mathrm{m}$ ) "cellophane-like" layer of preretinal cellular elements that forms over inner the surface of the macula. It is generally a slowly progressive problem (months) that affects the central vision by distorting the retinal surface thereby leading to the perception of visual blur and distortion. To improve vision, this membrane is surgically "peeled" off of the retina, the procedure is technically demanding and associated with significant surgical risk. A simple hook or microforceps is often used to engage and delaminate the pathological ERM. With this force-sensing tool, a customized hook was built and attached, as shown in Fig. 9, to determine whether the force sensor can be incorporated and used in a prototypical vitreoretinal surgical instrument. With essential small size and capability to provide force information, the novel tool has great potential to improve surgical training and performance in the retinal microsurgery. By adding the hook which translates the tool tip and consequently the point of force application, the calibration matrix needs to be regenerated with new force versus wavelength shift measurements. The new calibration matrix is

$K_{\text {hook }}=\left[\begin{array}{cc}-0.0172 & -0.0028 \\ 0.00638 & 0.01743 \\ 0.01083 & -0.01458\end{array}\right]$

To simulate epiretinal membrane peeling, we peeled and measured the forces generated during peeling of the inner shell membrane (ISM) of a 12 day old chicken embryo and a 3-week-old raw egg as shown in Figs. 10 and 11, respectively.

During the data collection the instrument was held close to $90^{\circ}$ to the membrane surface in order to minimize the force along the tool axis. The preliminary results show the various force profiles involved in membrane peeling collected and displayed in real time. This capability will allow us to monitor tool tissue interaction forces in membrane peeling
Fig. 10 Membrane peeling on the inner shell membrane in chicken embryo
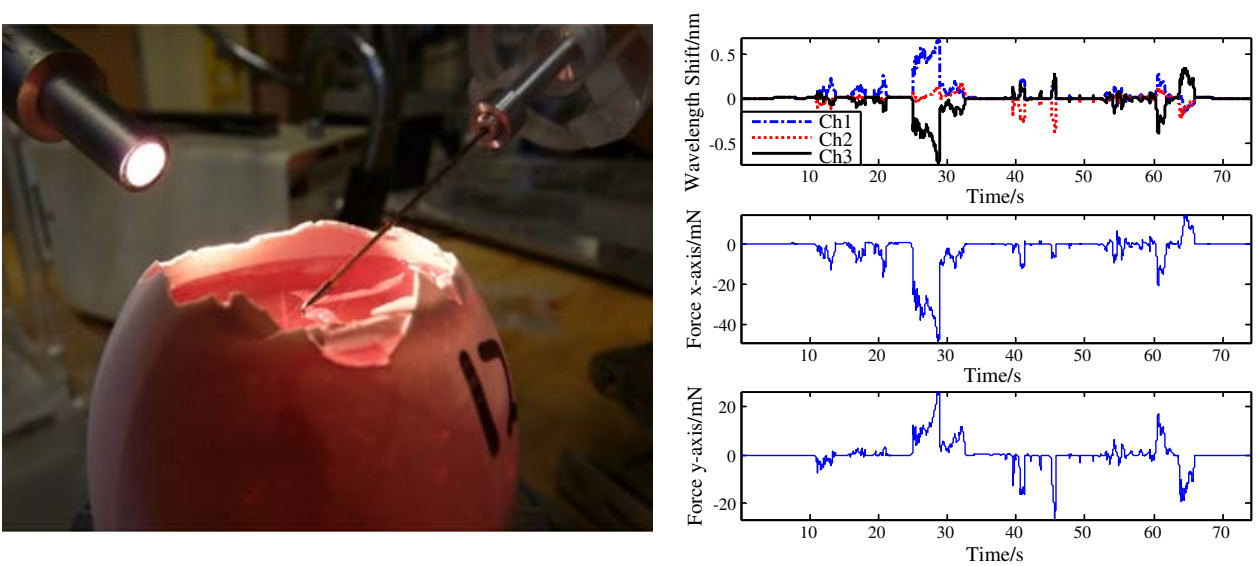
Fig. 11 Membrane peeling on the inner shell membrane of a raw egg
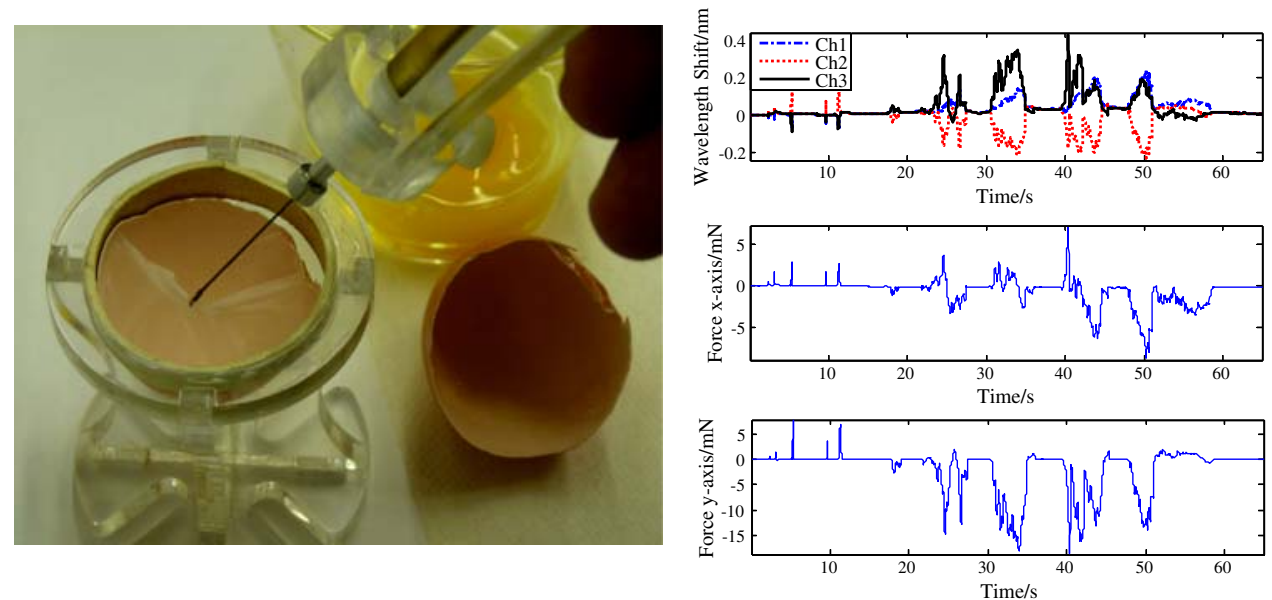

experiments. In addition, two experienced surgeons that tested both membrane models favored the raw egg as a good model for future experiments.

\section{Conclusion}

This paper has reported a new family of force-sensing microsurgical instruments. We have shown that placing FBG fiberoptic force sensors along the shaft of sub-millimeter diameter retinal instruments has sufficient force sensitivity to measure the extremely delicate forces associated with retinal surgery. Since the 10-mm long active element of the FBG sensor is embedded near the tip of the tool, which is completely inside of the eye ( $25 \mathrm{~mm}$ diameter), the tool-to-sclera interaction forces do not affect the sensor readings. Other advantages associated with our force-sensing approach include immunity to electrical noise, MRI compatibility, relatively easy to interface and sterilize. Its robustness and versatility due to size are ideal for microsurgical applications.

With the algorithm developed, we have successfully removed the temperature effect from the sensor readings. Only two sets of calibration are required to calculate the calibration matrix. A disadvantage is that the approach removes any forces related to axial strain. Though the sensitivity in measuring the strain in axial direction is much smaller compared to those along the transverse plane, the axial force measurement is still desirable for a complete a 3-DOF forcesensing tool. Further investigation of the necessity of the extra DOF and possible solutions is currently in progress.

With the 2 DOF sensing instrument presented here, we plan to begin a much more extensive series of studies to measure baseline tool-to-tissue forces during simulated and actual retinal procedures, as well as to develop sensory substitution and force-based robotic virtual fixtures for assisting the surgeon in carrying out these tasks. Examples of the former would include auditory or visual force cues (e.g., on a surgical microscope display). Examples of the latter include force servoing and force limiting behaviors to improve safety.

Acknowledgments This work was supported in part by the U.S. National Science Foundation under Cooperative Agreement EEC 9731478 , in part by the National Institutes of Health under BRP 1 R01 EB 007969-01 A1, and in part by Johns Hopkins internal funds.

\section{References}

1. Patkin M (1977) Ergonomics applied to the practice of microsurgery. Aust NZ J Surg 47:320-329. doi:10.1111/j.1445-2197.1977. tb04297.x

2. Gupta PK, Jensen PS, de Juan E Jr (1999) Surgical forces and tactile perception during retinal microsurgery, MICCAI'99. LNCS 1679:1218-1225

3. Iordachita I et al (2006) Steady-hand manipulator for retinal surgery. In: Proceedings of the MICCAI medical robotics workshop

4. Mitchell B et al (2007) Development and application of a New steady-hand manipulator for retinal surgery. In: Proceedings of the IEEE international conference robotics \& automation. Rome, pp 623-629

5. Okamura AM (2004) Methods for haptic feedback in teleoperated robot-assisted surgery. Ind Robot 31:499-508. doi:10.1108/ 01439910410566362

6. Zhou Y, Nelson BJ, Vikramaditya B (1998) Fusing force and vision feedback for micromanipulation. In: Proceedings of the IEEE international conference in robotics \& automation, Leuven, Belgium, pp 1220-1225

7. Kim K, Sun Y, Voyles R, Nelson B (2007) Calibration of multi-axis MEMS force sensors using the shape-from-motion method. IEEE Sens J 7(3). doi:10.1109/JSEN.2006.890141

8. Menciassi A, Eisinberg A, Scalari G, Anticoli C, Carrozza MC, Dario P (2001) Force feedback-based microinstrument for measuring tissue properties and pulse in microsurgery. In: Proceedings of the IEEE international conference robotics \& automation, Seoul, Korea (South), pp 626-631

9. Berkelman PJ, Whitcomb LL, Taylor RH, Jensen P (2003) A miniature microsurgical instrument tip force sensor for enhanced force feedback during robot-assisted manipulation. IEEE Trans Robot Autom 19(5):917-921. doi:10.1109/TRA.2003.817526 
10. Berkelman PJ, Whitcomb LL, Taylor RH, Jensen P (2000) A miniature Instrument Tip Force Sensor for Robot/Human Cooperative Microsurgical Manipulation with Enhanced Force Feedback. In: Medical Image Computing and Computer-Assisted Interventions, Pittsburgh, pp 897-906

11. Jagtap D, Riviere CN (2004) Applied force during vitreoretinal microsurgery with handheld instruments. In: Proc 26th IEEE engineering in medicine and biology conference (EMBS), San Francisco, pp 2771-2773

12. Hill KO, Meltz G (1997) Fiber Bragg grating technology fundamentals and overview. J Lightwave Technol 15(8):1263-1276. doi: $10.1109 / 50.618320$ 